Article

\title{
CRISIS: A System for Risk Management
}

\author{
Erica Y. Sanchez ${ }^{1, *}$ and Alejandro A. Acquesta ${ }^{2}$ \\ 1 Centro de investigaciones del Medio Ambiente (CIMA), Fac. Ciencias Exactas, UNLP, Argentina \\ 2 Departamento Modelado y Manejo de Crisis, CITEDEF, Argentina; \\ E-Mail: aacquesta@citedef.gob.ar
}

* Author to whom correspondence should be addressed; E-Mail: yaninasanch@gmail.com;

Tel.: +54-221-422-9329; Fax: +54-221-422-9329.

Received: 26 October 2012; in revised form: 12 November 2012 / Accepted: 30 November 2012 /

Published: 12 December 2012

\begin{abstract}
In a situation of an unexpected catastrophe, uncertainty and demand for information are constant. In such a disaster scenario, the CRISIS system arises as a tool to contribute to previous coordination, procedure validation, exercise execution, a good and frequent communication among organizations, and weakness and threat assessment for an appropriate risk management. It offers a wide variety of tools for online communication, consultation and collaboration that, up to this day, includes cartography, tasks, resources, news, forums, instant messaging and chat. As a complement, mathematical models for training and emergency management are being researched and developed. For Argentinean society, it is a necessity to switch from the current handcrafted, bureaucratic emergency management method to a decision-making management model. Previous coordination, exercise execution, a fluid communication among institutions, and threats and weaknesses assessment are required for a proper risk management. With that goal in mind, it is important to reduce confusion, avoid the duplication of efforts to fulfill the same tasks, and have access to a complete vision of the situation, generated from the data of all the organizations taking part. The CRISIS system is a secure web application, accessible to every node in a network formed by the organizations which have complementary responsibilities during prevention and response. It offers a wide variety of tools for online communication, consultation and collaboration that, up to this day, includes cartography, tasks, media (organization and resources), news, forums, instant messaging and chat. As a complement, mathematical models for training and emergency management are being researched and developed. Currently, there are toxicological and epidemiological emergency models available. The present paper analyses, from the perspectives related to
\end{abstract}


risk management for emergencies and disasters, the strengths and weaknesses of the CRISIS system to be used for prevention, response and recoveries in the case of a catastrophe.

Keywords: CRISIS system; risk management; emergency; disaster

\section{Introduction}

Emergency and crisis situations demand an effective interaction among multiple organizations with very different cultures, under the pressure of dealing with an event in evolution. Also, they demand the coordination for the usage of scarce material resources available, the management of consumables and goods donated by the general population for the people affected, and the organization of the actions to be carried out by the human resources available in response to the situation, with the highest synergy possible. It is necessary in such situations to manage and assess the information relevant to both the aforementioned aspects and the evolution of the original phenomenon, taking into account that this information is never absolutely timely, complete or accurate. In order to achieve this and consequently obtain efficient responses to emergency situations, a close coordination and collaboration with other sectors is also required to ensure that needs are fulfilled, efforts are not unnecessarily multiplied, resources are used optimally, and the medical care quality is appropriate [1]. On the other hand, not knowing the evolution of a phenomenon and its potential impact on the population may cause undesirable situations such as an inappropriate use of resources (which is a concern in developing countries where resources are limited) and poor decision making, among others.

We agree with Chen et al. [2] that there are four risk preference factors:

(A) The overall organizational risk culture.

(B) Risk management policy.

(C) Risk identification.

(D) Risk analysis, evaluation, treatment, and monitoring.

As stated above, we understand that a system capable of coordinating the parties and accurately representing the phenomenon can greatly contribute to manage the emergency and to meet the goals listed in the previous paragraph.

Although the CRISIS system is currently being used in a national scope by the Argentinean Department of Defense, it was designed to adapt to different working methods. Its design was influenced by the decree no. 1250/99 [3] for the creation of the Sistema Federal de Emergencias (SIFEM, Federal Emergency System), but it was intended to go beyond the scope of an information and alert system by including transversal coordination capabilities, information interoperability, task tracking, protocol activation and a collaborative geographic environment.

On the other hand, this tool is still in development, so certain functions are not yet available, only envisaged. Whenever an application capability that has not been deployed yet is referenced, it will be marked as "future" (f), and its study will be limited to those aspects already planned to be included. 
This work is, therefore, based on the experience obtained from technical research made by the Departamento de Modelado y Manejo de Crisis del Instituto de Investigaciones Científicas y Técnicas para la Defensa, Ministerio de Defensa de la República Argentina, between 2003 and 2012.

\subsection{Work Hypothesis}

The following ideas that gave a direction to the design of the CRISIS system arose from the needs of those who gave response in previous emergencies, the study of those emergencies, and the necessity of solving issues of coordination among organizations, and constitute the hypotheses for the development and ongoing optimization of the CRISIS system.

(i) Better information allows for better decisions: It is clear that the quality of the information is impaired when it is not complete, timely or truthful. The CRISIS system mainly offers tools to foresee risk situations, integrate information from one or many sources, and validate that information or qualify it as obsolete whenever that is the case. Therefore, we consider that any improvement in every aspect related to the quality of the information contributes to the betterment of the information as a whole, resulting in better decisions, see item (vi).

(ii) If something is not used during prevention, it will not be used during response: In the beginning of the development of the CRISIS system, the main goal was to give support during the crisis period immediately after the occurrence of an unfortunate event. But soon we came to understand that the organizations would not start to use a tool during a stressful situation, but they would resort to what is known and proven. This concept, together with the idea that prevention is as important as response, redirected the design of the system to support the integration of the competent institutions of the area, to have an operating network right since the planning, exercise execution, prevention and alert stages, in order to enable these organizations to manage the risks and create an environment where the coordination during response and recovery can follow naturally.

(iii) The solution should not be more complex than the problem: Analyzing emergencies and disasters, it is evident that events (and/or their effects) evolve in terms of scale, jurisdiction and severity. A situation that starts as a city issue may scale up to become a province-wide problem or even a nation-wide one, or it could just affect the nearest town. A situation that, in the beginning, arises as a sanitary problem may end up affecting transport, labor, education, production, etc. Also, the organizations involved in the response of a crisis may vary as time goes by, among those corresponding to the city, the province or the nation, or those state or private. For that reason, the CRISIS system was designed to ease up the spreading of the network among those responsible, by joining preexistent networks and linking new organizations as the situation becomes more complex, and its later reduction once the scale of the problem diminishes. It would be a serious mistake to maintain a single network with thousands of nodes acting together in absence of a great emergency; it is better to keep smaller local networks in operation that can be linked whenever the situation requires it. 
(iv) In times of change, one has to be flexible: Right from the first interviews of representatives from different organizations, it was evident that their methods for information management were very different. There are organizations which handle their data in a public and transparent way, while there are others which allow outsiders very limited access to their information. Although the current international trends, which Argentina endorses, encourage laws to guarantee public access to information, there are still changes to be made to be able to work in that way in our area. Nevertheless, it would be a mistake to expect that the adoption of a new tool will mend and unify the working methods of all parties. The CRISIS system was designed to allow each organization to choose and set policies relative to their individual access system. In other words, each organization can decide who they share their information with and who not. As the culture of each organization changes, the tools in use should be capable of reflecting that change, allowing the users to share whatever they consider appropriate at any particular time.

(v) Methodology and technology in a cycle of feedback and evolution: The perpetual advances in technology and the evolution of the methodologies for risk management and response to disasters are not completely independent phenomena. We consider that each change in methodology brings about the technological challenge to comply with that change, and each technological advance gives way to new tools to be exploited by methodology. Since the close relation between both concepts speeds up this virtuous circle, the decision was to work in interdisciplinary teams and to encourage a selection of human resources with diverse trainings.

(vi) Communication must be efficient and transparent; blackboard architecture: The blackboard concept is used to propose a collaborative structured space that allows an efficient communication between members of a growing institutional network, given the fact that the number of interactions needed to access a certain piece of information is independent of how many participants there are.

The first paradigm of coordination is a formal and vertical communication. In an ideal bureaucratic structure, communications are inspired in efficiency and economy principles, structured information and programmed decisions. Communications are always formal and flow through the tree structure of the organizational chart in a systematic way. The chart is created to deal with repeated and known situations, so they can be logged in forms and be treated deterministically. This architecture has serious disadvantages when maintaining an efficient flow of information during an emergency.

(1) In a tree architecture there is a single path to reach one node from another, so any break in the chain makes communications impossible, and in the best case scenario communications must pass through all the nodes in the middle.

(2) During emergencies or disasters, information tends to be unexpected and not to be repeated from many sources, hence structuring its content and therefore its treatment becomes difficult.

(3) Due to the facts expressed in 1 and 2, the information is transmitted from one level to the next and has to be interpreted in each node of the chain of communications (see Figure 1). 
Figure 1. In formal, vertical communications, when unstructured information is transmitted, the quality of the information is usually impaired, like in children's broken telephone game.

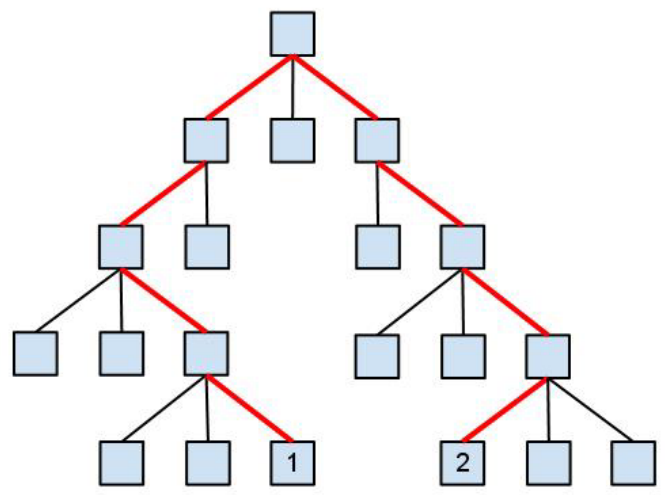

Therefore, the way information is handled in each node is critical, the transmission paths are not redundant, and when and if the information reaches its final destination, it does so with maximum delay and interpreted several times, which degrades the original data. In other words, as in a children telephone game, communications are inaccurate and slow.

To add to what has previously been learnt, our experience supports these facts: very often, coordination needs to rely on a less formal organization to ensure information will be validated later by the highest authority.

The second paradigm of coordination, an informal and horizontal communication, allows a direct contact among institutions, which shortens the time to reception, avoids intermediate interpretations of the information and multiplies the communication paths. Nevertheless, this approach poses a threat: the complexity of such a network with all the nodes connected with each other increases in a quadratic way as the number of nodes increases, as is shown in Figure 2. Therefore, in larger networks, each node should be linked only to a small neighborhood of other nodes, those with which they have a common working experience, those which have shared or neighboring jurisdictions, and, on occasions, those with personnel who have a personal connection.

Figure 2. In a network where each node is connected to all the others, the amount of links increases critically when the network has more than a few nodes.
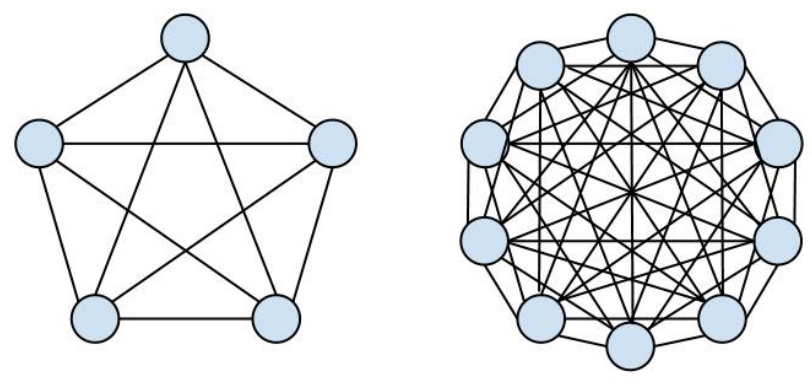

It is worth mentioning that on several occasions the informal and horizontal communication approach is efficient and sufficient, particularly to deal with situations such as those observed recently. Nevertheless, when there are changes in the personnel of the organizations, contacts and references get lost and therefore the communication dynamics is disturbed. On the other hand, there is a tendency to uncertainty, since news and consultation between two nodes that are not directly linked pass through 
complex and diverse paths. For that reason, valid information reaches certain nodes as almost rumors, since the original source is not readily known (see Figure 3).

Figure 3. In informal, transversal communications, a piece of news may travel an uncertain path and potentially not reach all relevant nodes. At the same time, the generator of any particular piece of information becomes uncertain.

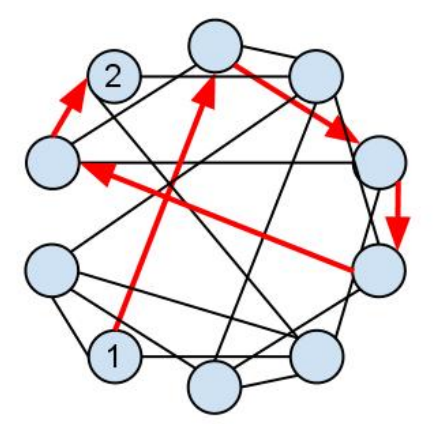

In recent years, many artificial intelligent models are proposed to solve all kinds of practical risk problems (see [4]). The DMMC proposal for approaching this matter is inspired in a multi-agent system coordination technique that arose from artificial intelligence and is applied to communications among people: the blackboard architecture. This approach can be understood as the conceptualization of the following metaphor: 'A team of experts tries to find a solution to a common problem. Since the matter is so complex and cannot be tackled by any one of them individually, these experts must cooperate by coordinating their tasks. For that matter, they gather around a blackboard in which they share their findings relevant to the problem resolution. They look at the blackboard and, when any of them considers they can make a contribution to the resolution, they ask for a piece of chalk and write it down on the blackboard. In the meantime, they remain still. This process ends when an expert determines that the information on the blackboard is a solution to the problem'. Although this definition belongs to the realm of artificial intelligence [5,6], this approach is applicable to an efficient coordination among people.

Figure 4. Using blackboard architecture, effective communication takes only two steps, and the information becomes a single unit in plain view for everyone.

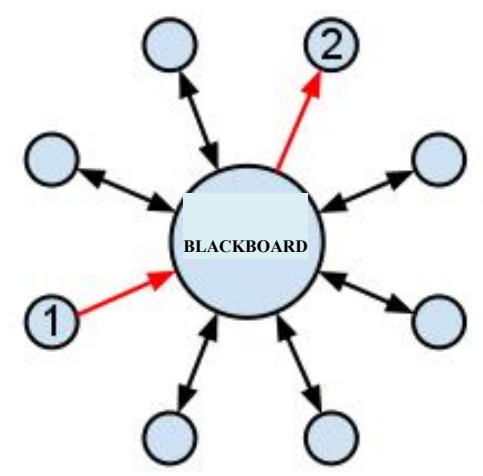

As shown in Figure 4, if all the nodes in an organization network have access to the post of news and contributions in a shared data space, and at the same time they can consult all the information posted in that space before making a decision, the total number of communication steps before making 
an informed decision is 2, notification and consultation, irrespective of the number of nodes involved. As a result, the information is more timely (since it is available sooner), more complete (since there is access to all shared information irrespective of its source) and more truthful (since there are no interpretation steps between those who generate the information and those who receive it).

\subsection{Related Work}

As a result of a comparative analysis with other tools used in emergency situations, it is observed that while the Sahana tool [7] allows public access to search and report missing people in a catastrophe, the CRISIS system provides access to the institutions involved in the risk and emergency management. In other words, the Sahana tool focuses on communication with the public while the CRISIS system works mainly in the coordination between the actors of the emergency.

Moreover, while the Sahana tool focuses on management of evacuation centers, their staff and supplies through a component called Mayon, the CRISIS system bring tools to command and control the tasks for disaster reduction, optimization of available communications and facilitate emergency management across the frequent scale changes in the situation. This is possible because the majority of their communications are independent of the number of nodes.

The model presented by Xiang Chen et al. [8], which was developed for evaluating the risk associated with evacuating the affected population in a disaster situation, shows a similar philosophy to the CRISIS system. Although they are both useful for scenario analyses and emergency management, the CRISIS system also considers the effect of interventions. A clear example of interventions can be seen in the following example: given a model that shows the probability of a road block in a city due to an evacuation, possible interventions could be: alter the direction of a street, turn a two way street in a one way street, etc.

Finally, a comparative advantage of the CRISIS system is that it is fully developed using open source software and open standards.

\section{CRISIS System Overview}

The CRISIS system [9] is a tool in the form of a web application, designed and developed to give a cooperative response to emergency situations; it offers solid and flexible communications, a more effective use of resources, a dynamic organization appropriate for the emergency, direct contact among all parties taking care of the emergency, the unification of a situational map, access to more and better information, and direct contact to information providers.

The CRISIS system is being developed at the Departamento de Modelado y Manejo de Crisis (DMMC, Department of Modeling and Crisis Management) of the Instituto de Investigaciones Científicas y Técnicas para la Defensa (CITEDEF, Institute for the Scientific and Technical Research for Defense), a division of the Argentinean Ministry of Defense. This department has been working since 2003 on the development of reliable computer tools for national-wide crisis management. During all these years, the DMMC has taken part in joined crisis management exercises with Chile, Bolivia and Peru, and in international seminars about technologies for crisis management. It has also collaborated in the monitoring of the Chaitén volcano eruption, the Irízar icebreaker fire, and the Mamoré river floods in Bolivia, and has given support to the Argentinean Health Department during 
the influenza A H1N1 epidemic alert, using the CRISIS system as the first national defense network for emergency management.

Since May 2010, the armed forces have been using the CRISIS system as a coordination tool to give support in civil defense operations. This system was also used during the recent earthquake in Chile for the coordination of the Air Force mobile hospital unit in the Curicó province.

Recently, the CRISIS system has been used in the Integración 2011, a joined exercise, in which Argentina, Bolivia and Peru took part, with a special participation of the province of Salta and the city of Salta.

Currently, its adoption is being contemplated for the Union of South American Nations (UNASUR) council of defense, the Argentinean chancery crisis room, the Vicente López city council, and the governments of the Gral. Alvear, Malargüe and San Rafael departments in Mendoza.

\subsection{Tools Available}

(I) Cartography: Allows the consultation and interaction of geographic information, generation and spreading of risk maps, subscription to external geographical information and view of GeoRss (geographically located alerts). In future stages, the monitoring of fleets using livefeeds (f) will be available. See Figure 5.

Figure 5. Cartography application of the CRISIS system showing volcanic ashes (SMN).

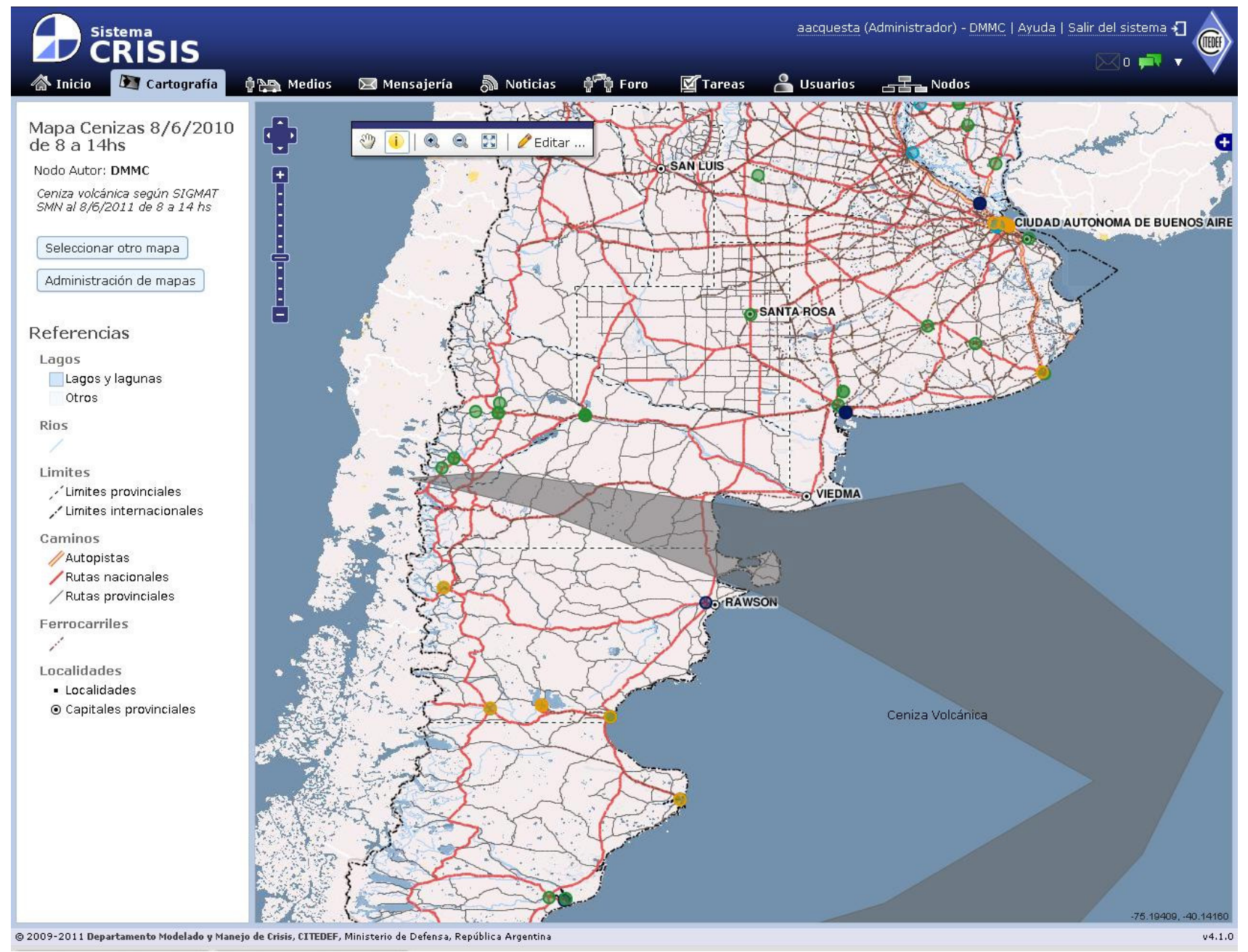


(II) Tasks: Allows the logging, assigning and tracking of the response activities, document attachment to each task, and task monitoring. See Figure 6.

Figure 6. Tasks application of the CRISIS system; picture taken from the Integración 2011 exercise.

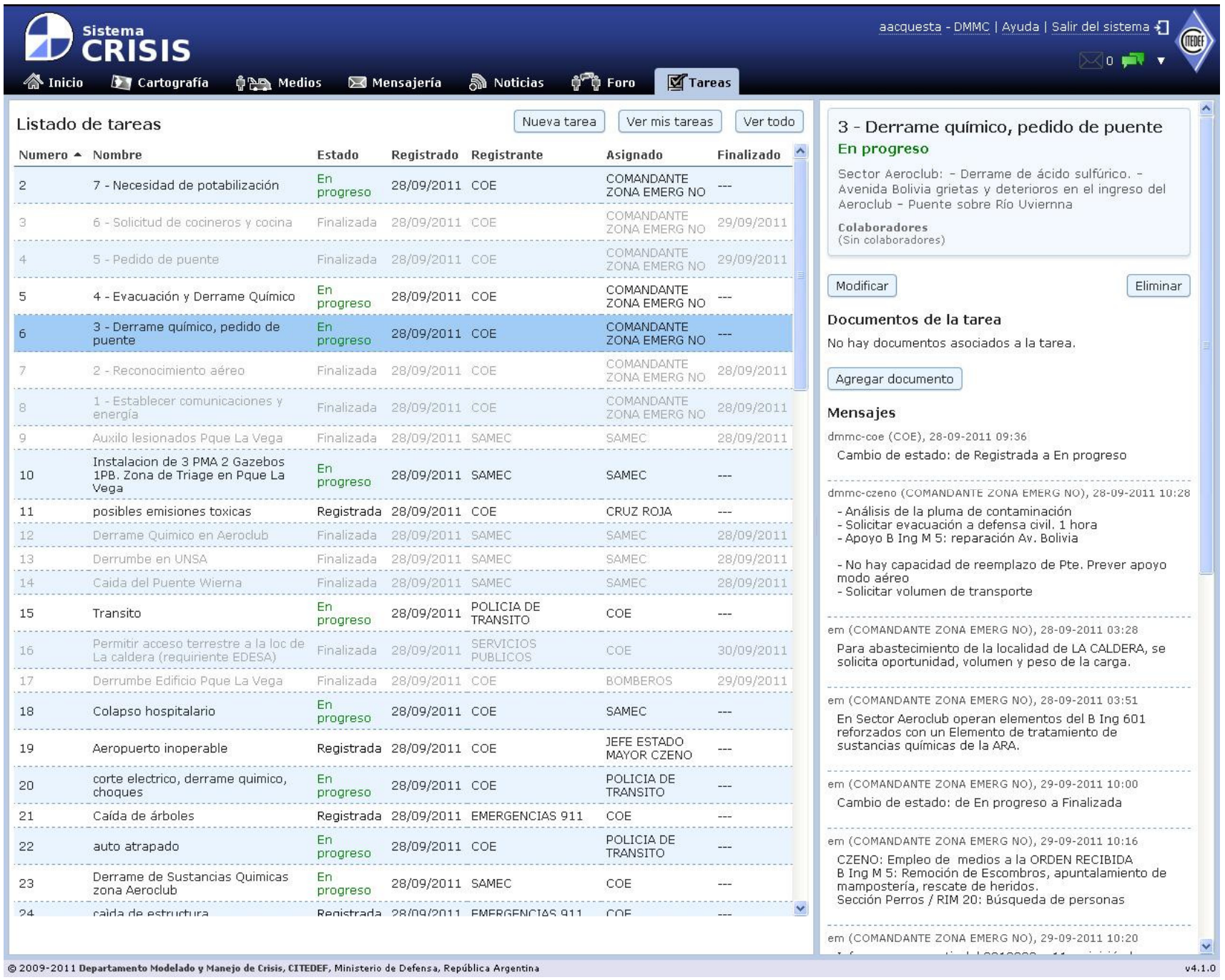

(III) Media: Allows the registration of the internal organization of an institution, keeping location, dependencies and people in charge relative to human and material resources available for operations. It also allows for the search for resources from internal and external sources, with their locations and dependencies, in order to ease the management of multi-agency operations. See Figures 7 to 9. 
Figure 7. Media application of the CRISIS system.

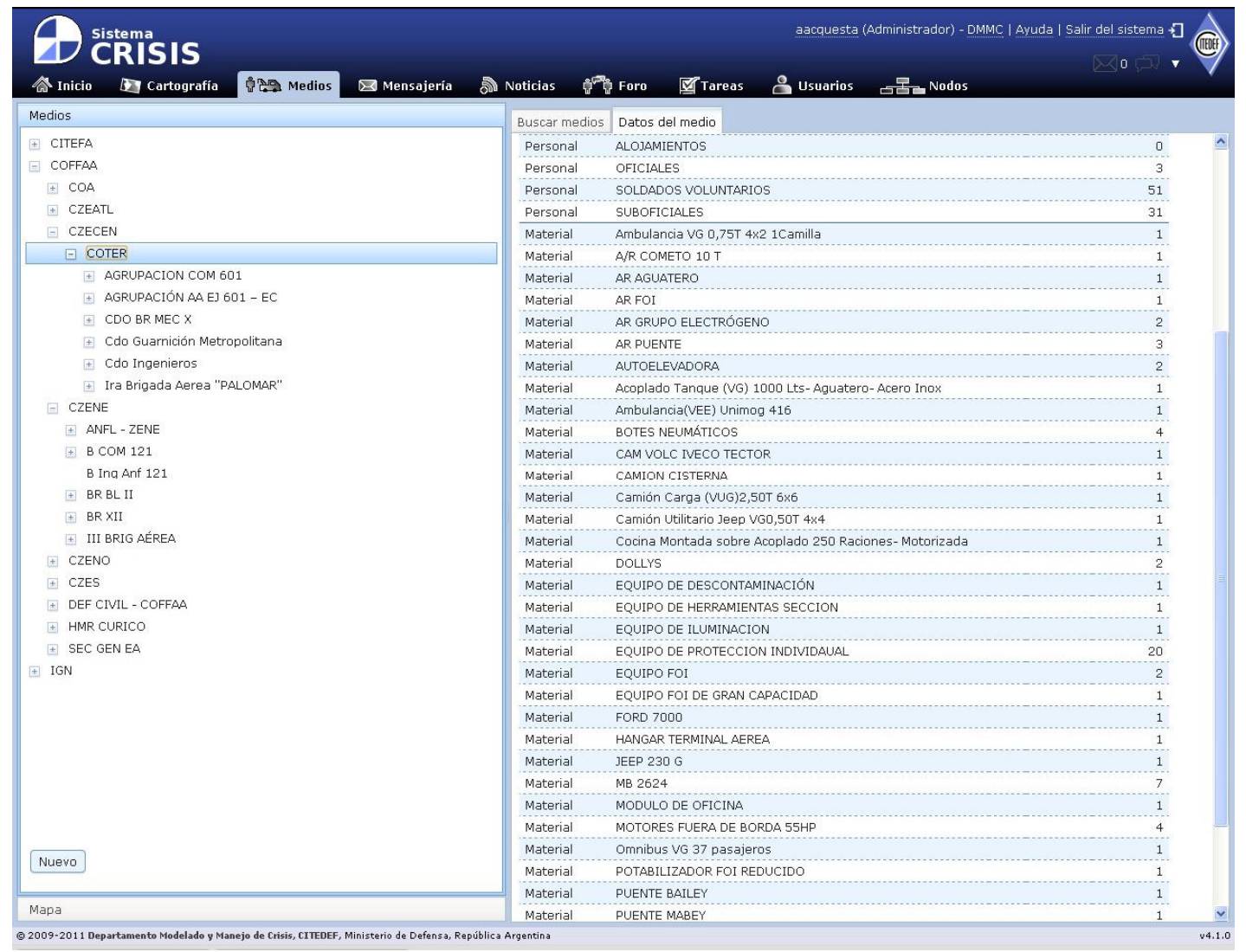

Figure 8. Media application of the CRISIS system; the map view shows the resources on the map.

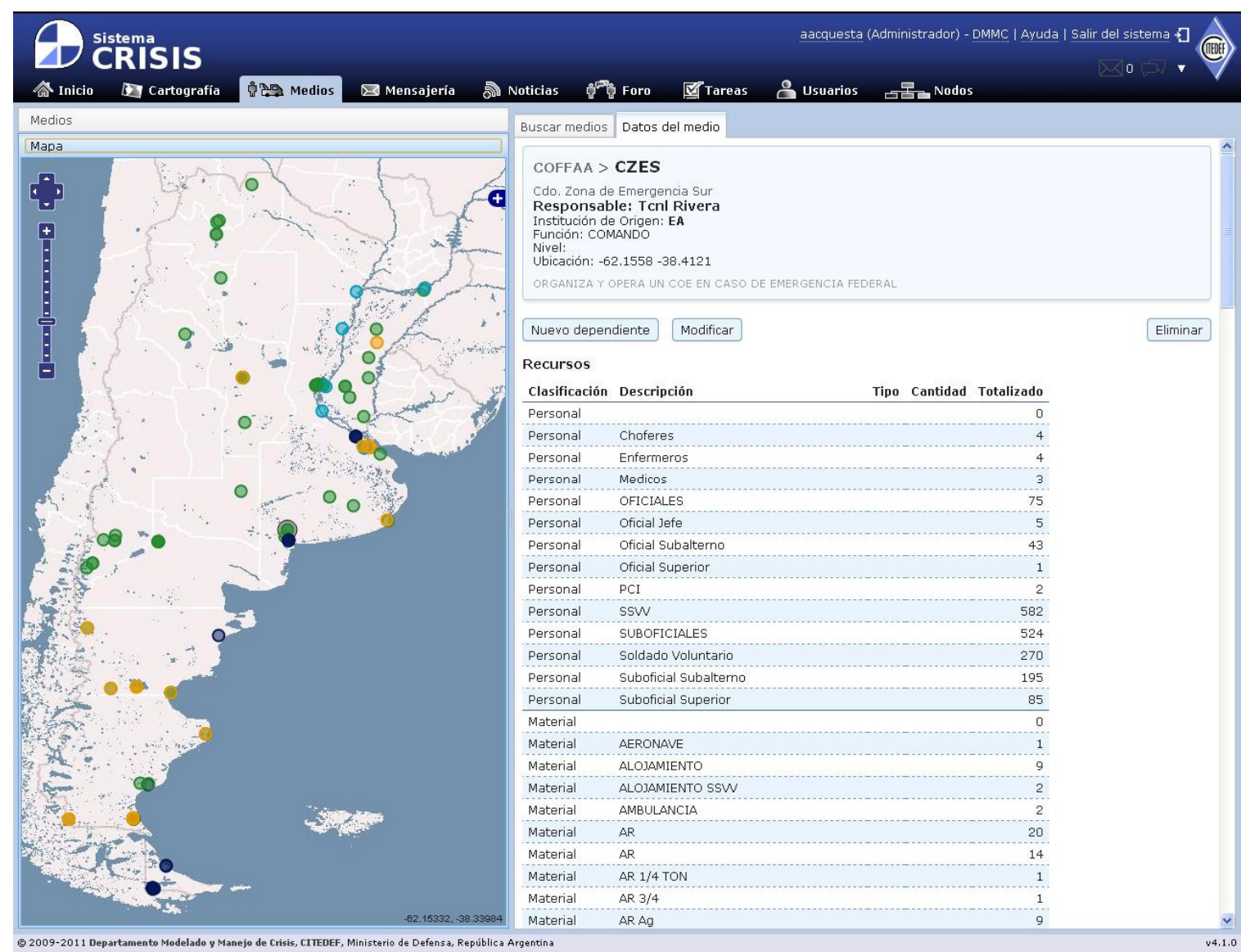


Figure 9. Media application of the CRISIS system; resource search on the map.

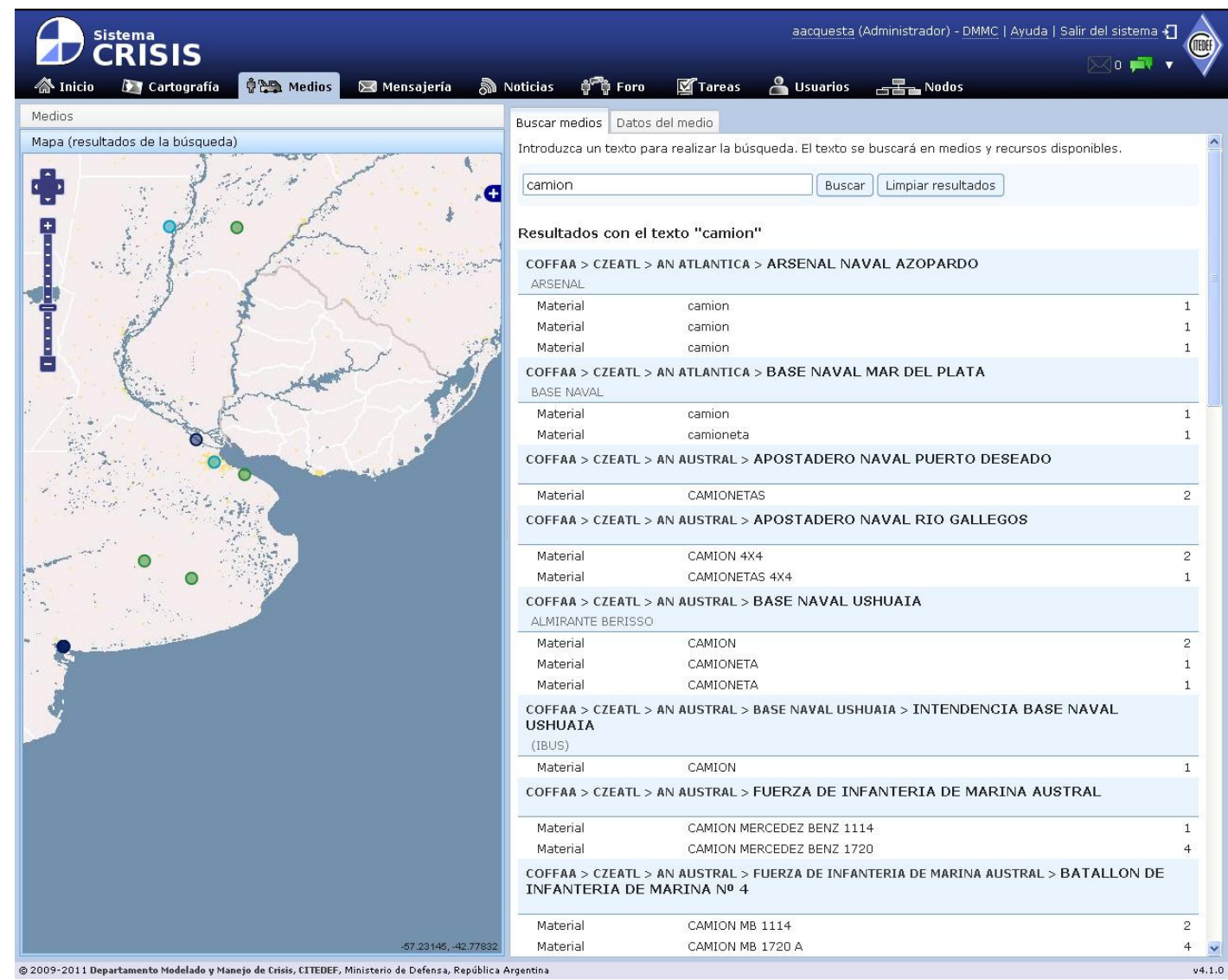

Figure 10. News application of the CRISIS system; heat spot alert list (CONAE).

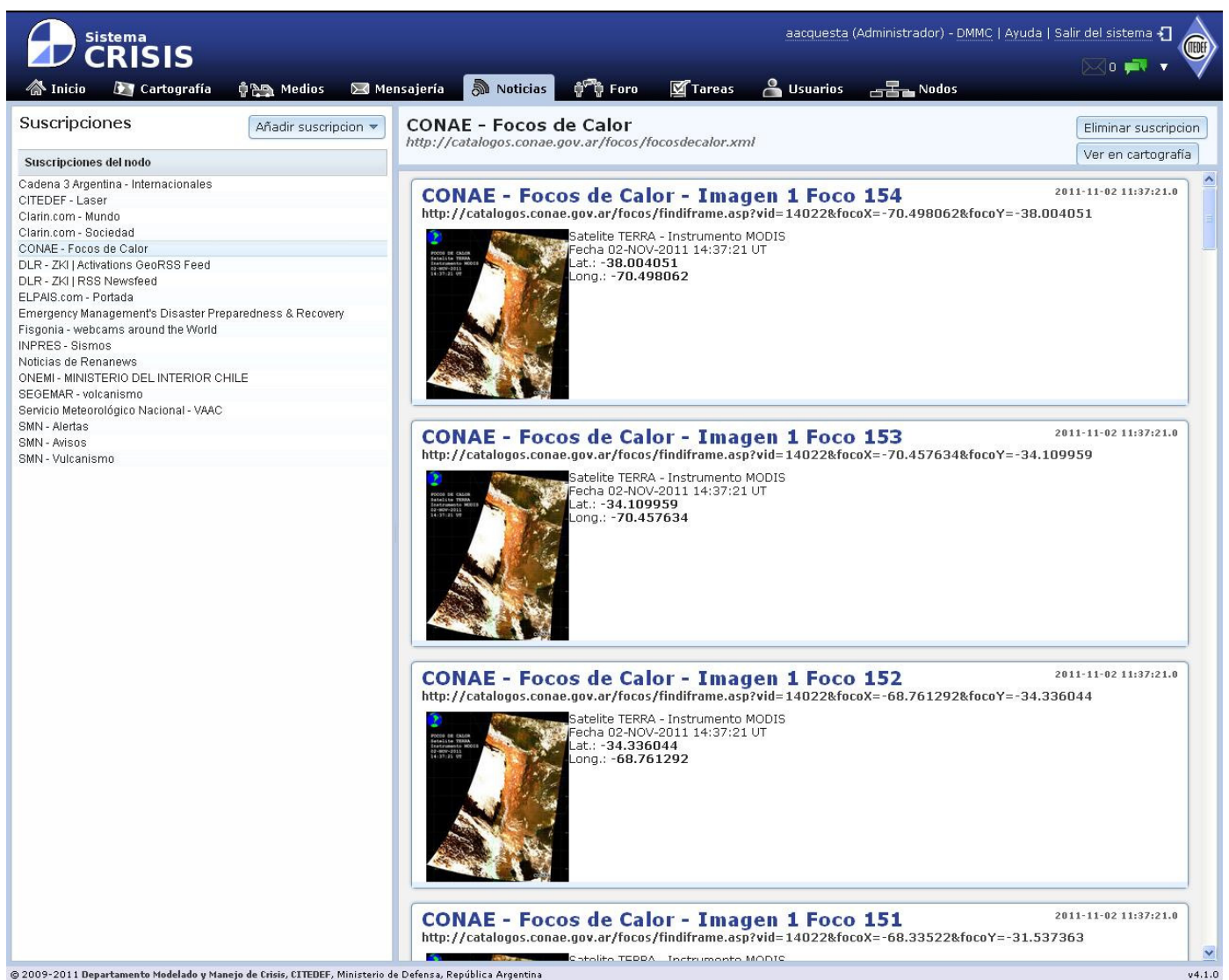


Figure 11. News application of the CRISIS system; map view of the heat spot alert list (CONAE).

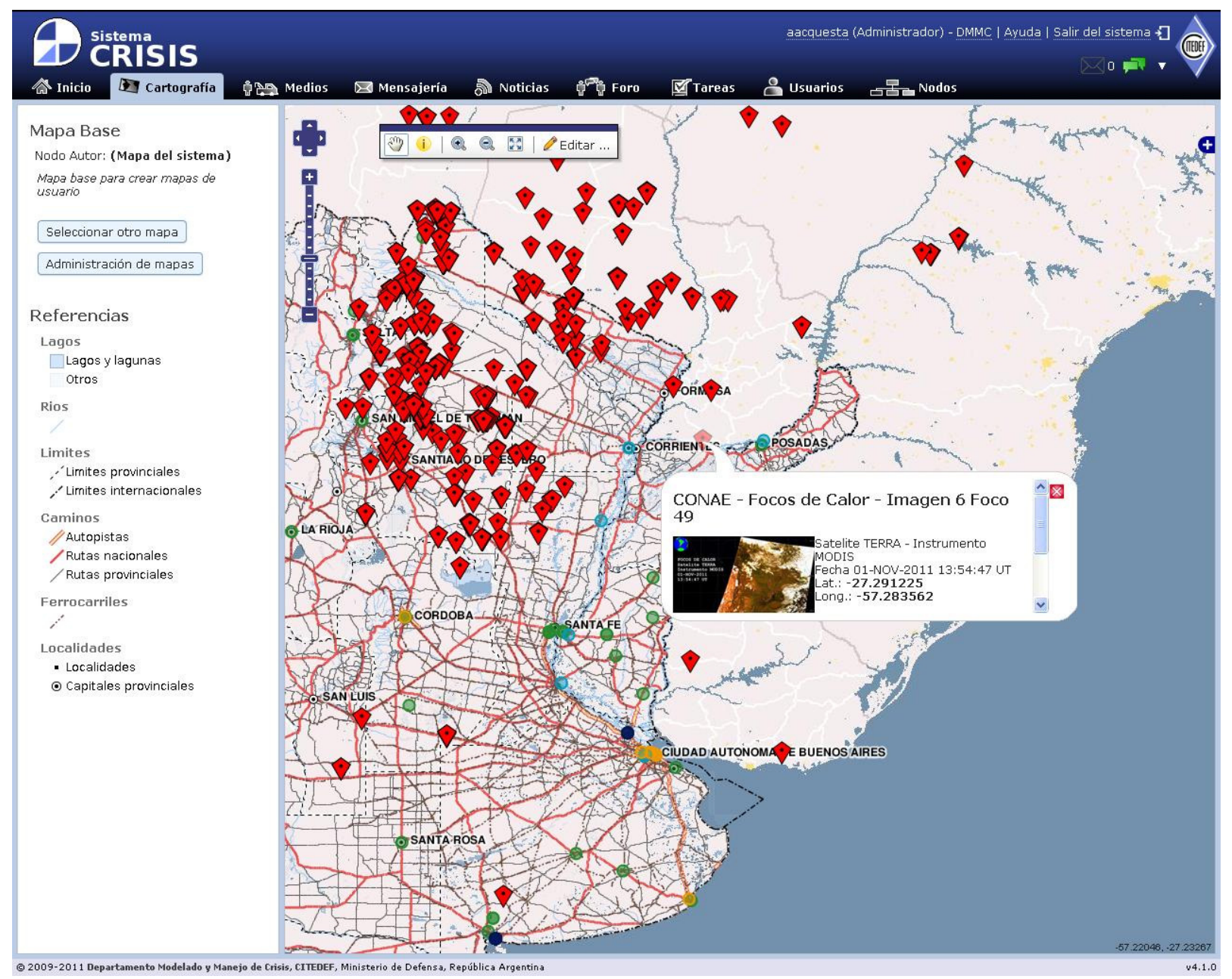

(IV) News: Organizes the subscription to sources of news and alerts about phenomena using Rss and GeoRss, and allows for the tagging of a location related to each feed entry onto the cartography. These subscriptions are open and each node may add the information sources it usually works with, share them and even associate its own information sources (press exploitation, internal reports, blogs, etc.). See Figures 10 and 11.

(V) Forum: Allows the continuous participation of all nodes in the network for help, discussion, response and coordination.

(VI) Instant messaging: Allows written communications between nodes for an effective, formal, and virus- and spam-free internal coordination.

(VII) Chat: Allows online, informal communication among system users, giving access to contact information of each user.

(VIII) Blackboard: (In development) Allows the management of a virtual board associated to the node or office, so that dynamic or static documents can be posted to be available for all the users in the node, other nodes, or even the whole community. Through this tool, it will be easy to give recommendations for the community, share information among nodes, monitor dynamically evolving information (lists of people evacuated, injured, deceased; list of resources needed), have access to critical documents from any location and avoid the spread of files with information that quicly becomes obsolete. 
(IX) Integration with mathematical models: Works on the assumption that the knowledge of different experts about different phenomena can be systematized for response planning, risk assessment, exercises, and aids in decision making during an emergency. To satisfy these requirements, models must:

- Use geographical and/or tabular information without major changes.

- Offer a possible response, quantifying the phenomena and potential effects on people, goods, infrastructure and environment with geographical references, in a standardized format.

- Obtain simulation results in a short time, which are very useful for real-time emergency response. It must be noted that there is a relation between the degree of detail of a simulation model and the simulation time; therefore the models that offer a great degree of detail about the phenomenon will be very useful in the planning stage.

- Evaluate disturbances during their occurrence, in order to consider the changes in atmospheric conditions and the effects of control interventions, among other issues.

In regard to this last tool, the CRISIS system supports any model for the simulation of natural or anthropogenic phenomenon, but provided there are a reliable database and a clear scientific understanding of the event, it is appropriate to use a numerical model [10,11].

Meanwhile, Chen and Tseng [12] conclude in their work that the stochastic cost-benefit-risk analysis model for enterprises is more suited to achieving the three equivalent goals of natural disaster risk management, i.e., maximizing shareholder wealth, share price, and firm value.

The DMMC intends to offer a more thorough comprehension of the scenarios to study and, in turn, a more efficient protection of the affected population's health, the environment and the affected assets. The CRISIS system and the simulation models can be used independently or together (f). Through their interaction, we intend to achieve more versatility, ease of use, and optimization.

The CRISIS system and its mathematical models are in continuous development, and its new functionalities are added as its users identify new needs, and time and resources allow for their integration. This feature has had a positive impact on the improvement of the application, which has been an ongoing process since 2003.

Finally, the models for earthquakes, such as the proposed models by Tseng and Chen [13], and Chen and Tseng [12]; the models for debris flow, such as the proposed models by Calvo and Savi [14], Chen et al. [15], Archetti and Lamberti [16], and Liu et al. [17] can usefully be incorporated into the CRISIS system.

\section{CRISIS System for Risk and Emergency Management}

According to its features, the CRISIS system offers a range of tools for different kinds of users connected through the Internet. This system makes access to information for planning and response easier, enabling contact with the relevant authorities to ask for and offer resources, and simplifying the planning and response to emergencies and disasters. The application provides a unified situational map, consultation and handling of geo-referenced information, non-restrictive data structures, support for early alerts, communication among participating organizations, access to external sources of information, and a user-friendly interface [18-23]. 
The CRISIS system manages the feedback from the real/regional data during the emergencies through three channels:

(1) Subscription to web services (rss, georss, cap) from different official information providers, allows monitoring the evolution of the different phenomena (meteorology, fires, floods, volcanic eruptions, etc.).

(2) Subscription to web services (rss, georss) from news agencies clarifies which information (correct or otherwise) is available to the public. This also enables deciding when, what and how to inform the public of the situation.

(3) The tasks component allows registration of all the needs surveyed from the terrain, plan the response actions, give orders and receive feedback of progress. It constitutes a soft interagency command and control system.

Moreover, the system allows incorporating new actors in case the emergency escalates. It is designed so that multiple response networks can be federated into one, whenever the situation requires.

Next, we explain the scope of the CRISIS system for an integral emergency management.

Prevention: It enables to practice, analyze and spread the response procedures; to form a decentralized database of resources available for potential crises; to coordinate and inform about actions tending to prevent and/or manage a risk; to assess disaster scenarios; to share risk maps; to keep a record of the organized means, and the human and material resources available; to log and monitor the prevention, training, planning and preparation tasks; and to inform and monitor the occurrence of harmful events.

Response: It enables to spread alerts; to start up response protocols, and to track the response tasks. It also eases up the interoperability among different sources of information, encourages a transversal coordination using online collaboration, and guarantees access to a unified situational map, thus improving the quality of the information for making decisions. It updates the records of human and material resources used in the response, and records and exploits information about donations and volunteers offered.

Recovery: It enables to assess the response for the improvement of the processes applied, and to record and monitor the tasks tending to mitigate, rehabilitate and rebuild.

Finally, we present historical cases that illustrate how the CRISIS system can be used in prevention, response and recoveries:

Prevention: the system has been used in multiagency training exercises, where procedures were corroborated and mathematical models were used to evaluate the effect of different phenomena.

Emergency monitoring: it was used to monitor the support given by the Armed Forces to the government of Bolivia during de Rio Mamoré floods, the fire on the icebreaker Almirante Irizar, and the eruption of the Chaitén Volcano.

Alert, readiness and response: it was used during 2009 to interoperate information from different ministries, during the A-H1N1 epidemiological alert. The Ministry of Health parameterized a mathematical model for a flu pandemic, evaluating possible scenarios and analyzing the effect of different kinds of interventions.

Response and recovery: it is being used as a coordination tool between eight ministries participating in the Plan de abordaje Integral AH1, which aims to improve infrastructure and living 
conditions of emergency settlements in the city of Buenos Aires and the province of Buenos Aires. This plan will last for several months, in areas with poor access to health, nutrition, education, security, employment and transportation. These are the reasons why the activities focus more on recovery (reconstruction and rehabilitation).

\subsection{Features for Emergency Logistics}

Logistics is a system which includes the supply, transport, storage and distribution of resources, and the provision of support services, and in which the interrelation of its parts simplifies, speeds up and organizes the development of the emergency operations, through an optimized usage of the available resources [24]. As part of this system, it must be achieved that the resources required are appropriate for the kind of event or emergency happening, in the appropriate amounts, under the appropriate conditions, at a fair cost, and at the precise time and place.

\subsubsection{Resources and Organized Means}

The CRISIS system was developed based on the working methodology of the Joint Chief of Staff of the Argentinean Armed Forces. For the Armed Forces, an organized means is a military device with one responsible leader, a geographical location, a series of operating capabilities, human and material resources, and, in certain cases, other subordinate organized means.

Therefore, the responsible leader of an organized means may receive an order from their superiors, since they have operating capabilities to display on a determined land extension, and the authority required to accomplish the mission assigned.

Inspired by that working method and evaluating a possible military and civil use of our tool, a database of organized means was deployed, interoperated by all organizations taking part in the network. Each organization may use this tool to record the information about different units, their location, contact information of the person in charge, their function and level, and the human and material resources it has. The information uploaded to the database can be consulted by other organizations, but only modified by the one which provided it.

The tool allows the search of means, capabilities, or just resources, including their status, location, and organizations and people in charge to whom these assets must be requested. At the same time, it allows to view the hierarchical structure as reported by an organization and a complete summary of its resources.

That way, the tool enables a more agile organization of multi-agency operations, shortening the time of coordination among the people in charge of each resource.

The tool can also be used to record the organized means of organizations that do not have access to the system; in this case, the organization willing to offer them creates an entry for that means, associates it to a work link (f) and records the relevant information.

\subsubsection{Donations and Volunteers}

It is relevant to mention the exploitation of human and material resources that do not belong to the organizations taking part in the network. It is a well-known fact that good-willed volunteers can easily 
become victims due to lack of organization. It is also known that donations are frequently an issue, since organizations receive goods that are not needed and are not previously classified, and their storage demands efforts, security and the use of other resources, sometimes during long periods of time.

The Means tool allows the recording of volunteers and donations offered, stating information about their location, person in charge, and specifics the human and material resources offered, without the need to physically receive them. That way, they can be found through the search engine as they are needed, contemplating their appropriateness. Only at that time, the person in charge is contacted to set a delivery or collection.

\subsection{Features for Communications during an Emergency}

The CRISIS system offers communication capabilities among organizations through a network, thus forming a specific channel for coordination and information interoperation. The aforementioned blackboard architecture paradigm allows to critically reduce the number of messages needed to guarantee that all nodes are well informed.

On the other hand, the possibility of having alert and news channels and also setting a level of importance for each channel allows to focus the attention onto the response or risk management tasks, knowing that important information will trigger a sound alarm or even a text message to a cell phone (f).

Another aspect that is worth mentioning is that a level of access can be set for each piece of information (maps, tasks, means, etc.) posted by a node, thus allowing the deployment of a permission policy with the following classification:

- Private: it can only be viewed by the users of the node that generated it.

- Restricted: it can only be viewed by specified nodes.

- Shared: it can be viewed by all the nodes in the network.

- Public: it can be viewed by all the nodes in the network, and nodes in other networks, and is open to the whole population through the Internet.

\subsection{Feature for Disaster Scenarios}

According to the definition of the Asociación de Proyectos Comunales de El Salvador (PROCOMES, Association for Community Projects of El Salvador), a risk scenario is a representation of the interaction of different threats and weaknesses set in a particular time and place. That scenario must represent and identify the damages and losses that may arise in the case of a disaster. Therefore, to generate a risk scenario, it is necessary to assess those threats and weaknesses, establish the possible risks accurately, and clearly identify those potential damages and losses. This thorough and complete representation of a risk scenario is a complicated task, since the weaknesses of a populated area can be both diverse and complex.

The assessment and diagnostics of such scenarios affect in a great amount the planning in advance for the potential occurrence of disasters and therefore the appropriate response to them. The evaluation of the weaknesses and capacities allows ensuring that the response in the case of a disaster will be effectively aimed to the people most in need, with a right to receive it, and without any bias. In order to achieve it, it is necessary to have a precise comprehension of the local context and of the way a certain 
crisis will affect specific groups of the population in different manners due to their preexistent inherent weaknesses [25].

In prevention and mitigation stages, the application of models to the evaluation of scenarios can contribute in a dynamic way to the community plans for the prevention and mitigation of disasters through a description of the risk or threat, and the consequent representation of the disaster scenario that may arise.

Typically the early hours after the occurrence of a disaster have the highest degree of uncertainty and, at the same time, are the most decisive to save lives and assets. To help in the decision-making process during that period, the mathematical models are very important in the CRISIS system [26-31].

On the other hand and as mentioned in previous sections, as a typical characteristic, the models representing a response in real time during a disaster describe fairly simple scenarios, since it is a requisite that the simulation results be obtained in a period shorter than the real evolution of the phenomenon. Nevertheless, for a first description of the situation, a quick decision making and damage assessment during the early stages of the phenomenon, the data amount provided by this kind of model is more than enough.

In any of the abovementioned stages, the simulation of the phenomena through mathematical models is intended to identify the population, dwellings and other infrastructure exposed to those phenomena, and at the same time to identify the causes of risk, thus contributing to the creation of comprehensive community plans that include every measure needed to know and counteract those risks, and to give an appropriate response in the case of an emergency or disaster with the aim of trying to reduce their potential impact on the community.

During a disaster, knowing how many people are potentially affected and to what extent in an appropriate time frame gives the decision makers the tools to reach conclusions concerning, among others, the time available to evacuate those who will be affected in the near future by the phenomena, the amount of people who will need immediate medical care according to the extent they are affected, and the logistics that will be necessary to respond to the emergency. Any detail that may contribute to the assessment of weaknesses, response capacity and threats is greatly valuable for risk management.

Profiting from the advantages offered by the mathematical models is closely associated with the need for complete and updated population information for the scenarios assessed in order to obtain an appropriate representation of the situation. Again, it is relevant to emphasize the need to enhance, complete and update the databases required for the models, since their optimization is not enough if measures are not taken to improve the information available.

The error is handled differently in each simulated phenomenon because it is intrinsically dependent on each model. For instance, the toxicological model informs users about the uncertainty of the results through a minimum and a maximum estimate of the expected effects on health, providing a confidence interval. Its calculation includes intrinsic error of the toxicological indices used.

\subsection{Feature for Operations Continuity}

According to the operation continuity approach, both the DMMC activity and the organizations using the CRISIS system demand that access to the web application be continuously available as a critical service provided by CITEDEF. To date, our network links and supports military units across 
the country, but there are several proposals of use for the transversal coordination of every kind of organizations.

\subsubsection{Identification of Critical Services}

Since our tool is intended to support organizations responding to emergencies, it has to keep working even when other services become offline, so it is critical to be prepared for the worst case scenario: how to operate the CRISIS system when the situation affects it.

\subsubsection{Ensuring Hardware Availability}

The system consists of two mirrored servers with two mirrored disk units each. These servers must be located in different buildings (f) and their connectivity must depend on different providers. There are two other servers in use:

- a server for development, and

- a server for testing and training.

The possibility of including a server for mathematical models and one for open organizational networks is being considered (f).

\subsubsection{Ensuring Energy Availability}

The electric power that feeds the servers comes from a separate line from all the others in the building. Also, we have an electric generator with the capacity to feed the server room and our office in the event of a power cut for a long period of time. We have the necessary consumables available to work on the electric generator for up to a month per year.

\subsubsection{High Service Availability}

Lastly, we are working on a protocol to be able to put the system online from any computer connected to the Internet, and we are researching the deployment of distributed clustering of servers in order to guarantee access to the system as long as any server in the cluster is still operating (f).

\subsubsection{Ensuring Technical and Methodology-Related Support}

Since this is a communication and coordination system, support may be provided through the exact same system, and the whole network and each node may be administered from any computer (or even a portable PC, smartphone, etc.) with a connection to the Internet. For instance, during the influenza A H1N1 epidemic alert, the Argentinean Heath Department provided a cell phone as a complement of the system for $24 \times 7$ consultations. We have planned the acquisition of two smartphones for the remote operation of the system (f), to support the development of compliant interfaces as well as to enhance the smartphone user experience to access the system.

Also, we plan to deploy a remote operation protocol for those cases when it is required (f). 


\subsubsection{System Emergency Deployment}

Another situation to be prepared for, is what to do if the user suddenly lacks power and communications.

We had the chance to simulate such a situation in the Integración 2011 exercise, carried out in the Salta city from September 26 to 30. On such occasion a communication break was simulated. Generators were installed to feed the convention center where the emergency operations center (COE) was operating.

Our team brought a portable PC with the system server installed and all the information uploaded in the last update instance. The COE computers were connected to the system through a LAN network, with no access to the Internet, and the system was used for the coordination of all the response activities, in connection with the radio networks of the police, the SAMEC division for sanitary emergencies, the army, civil aviation, etc.

From our experience in the Integración 2011, we are writing a protocol for the system emergency deployment, which will allow us to put the system in operation during a loss of connectivity to the data network and from any point across the country. An important issue is that the computer with the server installed has to be physically transported to the required location, and therefore it takes hours to finally have an operating system.

\section{Evaluation}

Currently, the CRISIS system is being used for coordination of eight ministries, the province of Buenos Aires and the local authorities in the framework of the Comprehensive Approach AHI. This operation addresses improvements in health, education, shelter, water sanitation and social integration for six slums in metropolitan and suburban Buenos Aires. About $80 \%$ of the emergency information is processed by the CRISIS system. That translates into greater flexibility for coordination and a dramatic reduction of paper information.

Additionally, the staff from different agencies interacts better in the field and there is consistency in the decision making of various actors who previously have worked alone.

The latter is possible since there is a direct contact with the situation. Nevertheless, the decisions made behind a desk with partial information result in differences between each decision maker's perception of the situation.

Moreover, there are only a handful of institutions that actually work with interoperable information and have efficient information sharing protocols. This is why the information fed to middle and upper management levels is often lacking and of low quality. This also results in different perceptions from different decision makers.

Finally, people on the terrain perceive the situation first hand, in real time. Many times, the result and the business continuity will depend on the ability to create multidisciplinary teams on the field.

\section{Limitations of CRISIS System}

The CRISIS system requires its users to have internet access. The cartographic information must be digitalized in .shp format, or accessible through a wms service to be interoperable. It requires a 
minimum three hour training course for regular computer users. In order to adapt itself to different institutions, the system does not set one specific way of dealing with a situation. This is why it is important to analyze the information flow between institutions in order to achieve a successful interpretation. The interconnection between different servers is designed but not yet implemented; neither is sending alerts to mobile phones and external e-mails, nor the implementation of the common alerting protocol, for early alerts propagation.

\section{Conclusions}

In the event of an unexpected catastrophe, uncertainty and demand for information are constant. Those who generate the necessary information are neither the same actors who make the critical decisions nor those who will give assistance at the place of the emergency. Also, organizations usually do not have a complete knowledge of their own human and material resources available, let alone those pertaining to other organizations with which they have to interact during the crisis. It may also be the case that the information relative to resources exists, but is not available to the decision makers in a timely manner.

Another characteristic of this kind of situation is an apparent difference in culture and language among the organizations that makes communication difficult. However, we cannot leave aside the fact that this occurs in completely abnormal circumstances, when people fear for their lives and those of their beloved ones.

Moreover, national and regional decision makers need to understand the degree to which decision processes affect the capabilities of a region to absorb the impact of a disaster and to respond and recover within an acceptable envelope of both time and cost. Regions are interconnected and interdependent networks of technologies, businesses, organizations, infrastructures, and socio-political realities that require one another for continued efficient operation. The evaluation of regional capabilities, preparedness, and resilience is complicated by their interconnected and dependent nature as well as by their highly decentralized and distributed governance schemes [32-34].

In such a disaster scenario, organizations need to relate in a different manner than the regular one to give appropriate responses. Depending on the area affected, jurisdictions usually collide and there is no time for a political argument. Usually there are different pieces of information which do not make sense altogether and there are lives and assets in danger.

The CRISIS system, therefore, arises as a tool to contribute to previous coordination, procedure validation, exercise execution, a good and frequent communication among organizations, and weakness and threat assessment for appropriate risk management. Each organization connected to the CRISIS system network has its own attributes: some of them just provide primary information; then they make it available for the decision makers, who are typically government officers. That way, instead of resorting to their typical information channels, the decision makers consult this single, common space that acts as a 'big blackboard'. The decisions made become pieces of information that are in turn posted on that same board, to make them available for those who have to take action and direct the human resources for assistance; they will already know where to look up the relevant, accurate and updated information. That way, everybody can work with less uncertainty.

As conclusions in this paper, and in answering the goals set, it is evident that: 
- There is an apparent progress in technology developments relative to our area, and those developments bring about useful tools for disaster and risk management.

- The working methodology for risk management can also make use of these developments to fulfill their goals.

- The mathematical models can allow for the assessment of disaster scenarios in less time and more thoroughly.

It is also evident that:

- IT systems must be perfected taking the users into account in order to contrast those systems with each organization's work methodologies in place, so the users are the ones who validate the system features and make the tool their own.

- In many towns a change must be made in the working methods to be able to successfully deploy risk management strategies. We firmly believe that using a tool that provides permanent contact may help the growth process.

- For the mathematical models to be useful for those responsible for planning and response, they must have a simple interface which is permanently accessible.

We believe there is still much to be learned about the subject of disasters, but the understanding that this matter must be approached from several different disciplines and that technology can greatly contribute to risk management is a huge step forward. Lastly, it is important to mention that this process of continuous optimization of the CRISIS system has been put in place because we understand and affirm that technology and methodology are an evolutionary cycle that provides constant feedback in order to grow.

\section{Acknowledgments}

The authors acknowledge the support of the National Agency for Scientific and Technological Promotion of Argentina (PICTO 2004 11-18622), the National Council of Scientific and Technical (CONICET) and CITEDEF for financing the present study.

A.D. Acquesta is a student of the career of Doctor of Engineering from the Technological Institute of Buenos Aires.

We thank Romina L. Ferraro and Ing. Graciela Defeo for valuable discussions and help in the preparation of the final version of this article.

\section{Conflict of Interest}

The authors declare no conflict of interest.

\section{References}

1. Sphere Project. The Sphere Handbook: Humanitarian Charter and Minimum Standards in Humanitarian Response, 3rd ed.; Practical Action Publishing: Rugby, UK, 2011; pp. 63-87, ISBN 978-1-908176-02-8. 
2. Chen, C.W.; Liu, K.F.; Tseng, C.P.; Hsu, W.K.; Chiang, W.L. Hazard management and risk design by optimal statistical analysis. Nat. Hazards 2012, 64, 1707-1716.

3. Decreet 1250/99. Available online: http://www.disaster-info.net/PED-Sudamerica/leyes/leyes/ suramerica/ argentina /sistemnac/Decreto_1250-SIFEM.pdf (accessed on 1 June 2012).

4. Chen, T.H.; Chen, C.W. Application of data mining to the spatial heterogeneity of foreclosed mortgages. Expert Syst. Appl. 2010, 37, 993-997.

5. Rizo, R.; Llorens, F.; Pujol, M. Architectures and communication between agents. In Agentes Inteligentes: Sistemas Multiagentes y Aplicaciones; Skarmeta, A.G., Pujol, M., Rizo, R., Eds.; Alicante, España, 2002; pp.181-214, ISBN: 84-8454-182-7.

6. García Serrano, A.; Ossowski, S. Distributed artificial intelligence and multiagent systems. Inteligencia Artificial, Revista Iberoamericana de Inteligencia Artificial 1998, 1-12.

7. Sahana-Software Foundation site. Available online: http://sahanafoundation.org/ V (accessed on 15 August 2012).

8. Chen, X.; Kwan, M.; Li, Q.; Chen, J. A model for evacuation risk assessment with consideration of pre- and post-disaster factors. Comput. Environ. Urban Syst. 2012, 36, 207-217.

9. CRISIS system site. Available online: http://www.sistema-crisis.gob.ar/ (accessed on 15 August 2012).

10. Sanchez, E.Y.; Colman Lerner, J.E.; Porta, A.; Jacovkis, P.M. Accidental release of chlorine in Chicago: Coupling of an exposure model with a computational fluid dynamics model. Atmos. Environ. 2013, 64, 47-55.

11. US EPA, 2012, Support Center for Regulatory Atmospheric Modeling. Available online: http://www.epa.gov/scram001/ (accessed on 02 November 2012).

12. Chen, C.W.; Tseng, C.P. Default risk-based probabilistic decision model for risk management and control. Nat. Hazards 2012, 63, 659-671.

13. Tseng, C.P.; Chen, C.W. Natural disaster management mechanisms for probabilistic earthquake loss. Nat. Hazards 2012, 60, 1055-1063.

14. Calvo, B.; Savi, F. A real-world application of Monte Carlo procedure for debris flow risk assessment. Comput. Geosci. 2009, 35, 967-977.

15. Chen, S.C.; Wu, C.Y.; Wu, T.Y. Resilient capacity assessment for geological failure areas: Examples from communities affected by debris flow disaster. Environ. Geol. 2009, 56, $1523-1532$.

16. Archetti, R.; Lamberti, A. Assessment of risk due to debris flow events. Nat. Hazards Rev. 2003, 4, 115-125.

17. Liu, X.; Yue, Z.Q.; Tham, L.G.; Lee, C.F. Empirical assessment of debris flow risk on a regional scale in Yunnan province, Southwestern China. Environ. Manag. 2002, 30, 249-264.

18. Acquesta, A.D.; Sanchez, E.Y.; Jacovkis, P.M. CRISIS System for Risk Management and Emergencies. In Proceedings of the Second Congress SRA-LA-Regional Society for Risk, Bogotá, Colombia, May 2012; Muñoz, F., Eds.; Society for Risk Analysis Latin American: Bogota, Colombia, pp. 370-371.

19. Sánchez, E.Y.; Acquesta, A.D. El Sistema CRISIS para la Gestión de Riesgos. Master Thesis, Consejo Provincial de Emergencias e Instituto Provincial para la Administración Pública, PBA. La Plata, Argentina, 2011, (in Spanish). 
20. La Crisis Bajo Control. Miradas Al Sur, Year 3, 150th ed.; 3 April 2011. Available online: http://sur.elargentino.com/notas/la-crisis-bajo-control (accessed on 15 August 2012).

21. Acquesta, A.D.; Defeo, G.; Tarulla, F.; Giraldez, G.; Gonzalez, E.M.; Kuntscher, L.; Jacovkis, P.M.; Porta, A.A.; Sánchez, E.Y.; Filkensteyn, A. Computer Systems for Interagency Emergency Management. In Proceedings of the I Congreso Latinoamericano SRA-LA 2010: "El estado del análisis de riesgo en América Latina”, Santiago de Chile, Chile, August 2010.

22. Acquesta, A.D.; Sevilla, A.G.; Giraldez, G.; Defeo, G.; Tarulla, F.; Sánchez, E.Y.; Filkensteyn, A.; Porta, A.; Jacovkis, P. CRISIS System. In Proceedings of the XVI Congreso Argentino de Toxicologia, Puerto Madryn, Argentina, September 2009.

23. Acquesta, A.D.; Sevilla, A.G.; Giraldez, G.; Defeo, G.; Tarulla, F.; Sánchez, E.Y.; Filkensteyn, A.; Porta, A.; Jacovkis, P.M. CRISIS Project: Computational Models for Emergency Management in Real Time. In Proceedings of the II Congreso Argentino de la Sociedad de Toxicología y Química Ambiental (SETAC), Buenos Aires, Argentina, November 2008; Summary book.

24. FUNDESUMA. Manejo logístico de suministros de emergencia, Version 1.0. Curso manejo logístico de suministros de emergencia, San José, Costa Rica, 1999.

25. Communal Projects Association of El Salvador (PROCOMES). Manual de Conceptos básicos sobre gestión de riesgo y preparación local ante desastres. OXFAM, ECHO, 2008.

26. Sánchez, E.Y.; Gonzalez, E.M.; Colman, J.E.; Porta, A.A.; Jacovkis, P.M.; Acquesta, A.D. Model and Simulation of Regions Affected by a Chemical Incident, Ciencia y Tecnología Ambiental: Un Enfoque Integrador; Asociación Argentina para el Progreso de las Ciencias: Mar del Plata, Argentina, 2012; pp. 333-338, ISBN 978-987-28123-1-7 (in Spanish).

27. Sanchez, E.Y.; Acquesta, A.D.; Colman Lerner, J.E.; Porta, A.A.; Jacovkis, P.M. Analysis with DDC Coupled to Different Models of Dispersion in Air of Chlorine Releases. In Proceedings of the Second Congress SRA-LA-Regional Society for Risk, Bogotá, Colombia, May 2012; Muñoz, F., Eds.; Society for Risk Analysis Latin American: Bogota, Colombia, pp. 119-125.

28. Sánchez, E.Y.; Gonzalez, E.M.; Porta, A.A.; Jacovkis, P.M.; Acquesta, A.D. Simulation of a Chemical Incident with the Tool CFD-DDC: Emergency Response Planning in Cities. In Contaminación Atmosférica e Hídrica en Argentina; Puliafito, E., Ed.; Universidad Tecnológica Nacional: Mendoza, Argentina, 2011; pp. 257-268, ISBN 978-950-42-0136-6.

29. Acquesta, A.D.; Sánchez, E.Y.; Porta, A.; Jacovkis, P.M. A method for computing the damage level due to the exposure to an airborne chemical with a time-varying concentration. Risk Anal. 2011, 31, 1451-1469.

30. Sanchez, E.Y.; Acquesta, A.D.; Porta, A.A.; Jacovkis, P.M. Simulation of Chemical Accidents: Assessment of Exposure to Non-Stationary Models. In Proceedings of the I Congreso Latinoamericano SRA-LA 2010: "El estado del análisis de riesgo en América Latina”, Santiago de Chile, Chile, August 2010, (in Spanish).

31. Acquesta, A.D.; Sánchez, E.Y.; Porta, A.; Jacovkis, P. Método de cálculo del daño provocado por la exposición a un perfil variable en el tiempo, de concentración de contaminantes en el aire. In Proceedings of the II Congreso Argentino de la Sociedad de Toxicología y Química Ambiental (SETAC), Mar del Plata, Argentina, November 2008. 
32. Crowther, K.G. Risk-informed assessment of regional preparedness: A case study of emergency potable water for hurricane response in Southeast Virginia. Int. J. Crit. Infrastructure Protection 2010, 3, 83-98.

33. Crowther, K.G.; Haimes, Y.Y. Development of the multiregional inoperability input-output model (MRIIM) for spatial explicitness in preparedness of interdependent regions. Syst. Engineering 2010, 13, 28-46.

34. Stephan, R. National Infrastructure Protection Plan Represents Collaboration between Government and the Private Sector; The CIP Report; Zeichner Risk Analytics: Arlington, VA, USA, 2006; pp. 2-5.

(C) 2013 by the authors; licensee MDPI, Basel, Switzerland. This article is an open access article distributed under the terms and conditions of the Creative Commons Attribution license (http://creativecommons.org/licenses/by/3.0/). 